\title{
Unveiling the Features of Mercury-Associated Minimal Change Disease: Comparison with Primary Minimal Change Disease
}

\author{
Ai-bo Qin a, b, c, d, e Xiao-juan Yu a,b, c, d, e Su-xia Wang a, b, c, d, e, f \\ Fu-de Zhou a, b, c, d, e Ming-hui Zhao ${ }^{a, b}, c, d, e, g$

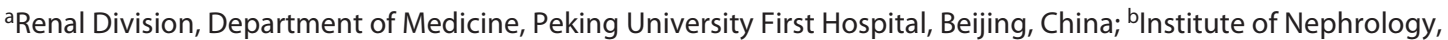 \\ Peking University, Beijing, China; ${ }^{C}$ Key Laboratory of Renal Disease, Ministry of Health of China, Beijing, China; ${ }^{d}$ Key

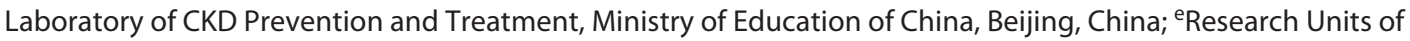 \\ Diagnosis and Treatment of Immune-mediated Kidney Diseases, Chinese Academy of Medical Sciences, Beijing, \\ China; ${ }^{\mathrm{f}}$ Electron Microscopy Laboratory, Pathological Center, Peking University First Hospital, Beijing, China; \\ gPeking-Tsinghua Center for Life Sciences, Beijing, China
}

\section{Keywords}

Mercury · Minimal change disease $\cdot$ Cosmetics $\cdot$ Nephrotic syndrome $\cdot$ Foot process effacement

\begin{abstract}
Introduction: Long-term exposure to mercury can cause minimal change disease. However, the current understanding of mercury-associated minimal change disease (M-MCD) is inadequate. To improve the understanding of $M-M C D$, this study retrospectively analyzed the clinicopathological, ultrastructural, and prognostic features of M-MCD, in comparison with primary minimal change disease (P-MCD). Methods: We retrospectively analyzed the clinicopathological data of 21 M-MCD patients and 21 P-MCD patients. Electron micrographs of glomerular capillaries were taken, and the foot process width (FPW) was measured. A receiver operating characteristics (ROC) curve analysis was performed to determine the optimum cutoff value of FPW that can differentiate the M-MCD from P-MCD. Results: M-MCD patients presented similar clinical and routine pathological characteristics
\end{abstract}

with P-MCD patients but had lower levels of FPW (935.0 [interquartile range (IQR) 853.7-1,176.7] $\mathrm{nm}$ vs. 1,403.2 [IQR $1,089.2-1,841.8] \mathrm{nm}, p=0.002)$. ROC curve analysis showed that FPW value below $1,385 \mathrm{~nm}$ might help to differentiate M-MCD from P-MCD (area under the curve of 0.787 , sensitivity of $94.7 \%$, and specificity of $52.4 \%$ ). For patients with MMCD, 77.8\% achieved complete remission after mercury detoxification monotherapy. Patients with M-MCD had a lower relapse rate than patients with $\mathrm{P}-\mathrm{MCD}(0 \mathrm{vs} .47 .1 \%, p=0.003)$. In addition, there was no significant difference in remission time between M-MCD patients treated with mercury detoxification monotherapy and those initially treated with immunosuppressive therapy (2.0 [IQR 1.0-6.0] months vs. 2.0 [IQR 1.5-2.5] months, $p=0.606)$. Conclusions: M-MCD patients showed similar clinicopathological features with P-MCD patients, but with less severe foot process effacement, suggesting different pathogenesis of these 2 disease entities. The treatment of mercury detoxification was highly effective for patients with M-MCD and can be considered as a primary choice in clinical practice.

(c) 2020 The Author(s)

Published by S. Karger AG, Basel karger@karger.com www.karger.com/kdd

Karger $\stackrel{\text { ' }}{5}$

BOPEN ACCESS
(C) 2020 The Author(s)

Published by S. Karger AG, Basel

This article is licensed under the Creative Commons AttributionNonCommercial-NoDerivatives 4.0 International License (CC BYNC-ND) (http://www.karger.com/Services/OpenAccessLicense) Usage and distribution for commercial purposes as well as any distribution of modified material requires written permission.
Fu-de Zhou

Renal Division, Department of Medicine, Peking University First Hospital 8 Xishiku St., Xicheng District

Beijing 100034 (China)

zhoufude1801@vip.sina.com 


\section{Introduction}

Mercury is a heavy metal with nephrotoxicity $[1,2]$. It has been known to cause proteinuria in humans as early as the year of 1818 [3]. Long-term exposure to mercury may cause different pathological patterns of glomerular diseases, such as minimal change disease (MCD) [4-6], membranous nephropathy (MN) $[7,8]$, and focal segmental glomerular sclerosis [9].

Mercury-associated minimal change disease (M$\mathrm{MCD}$ ) was reported to be the most common renal histopathological patterns of mercury-associated glomerulonephropathy $[6,10]$. The first case of proven MCD caused by mercury exposure was reported in 2006 [11], since then an increasing number of M-MCD cases have been reported [5]. Due to a lack of specific clinical manifestations and systemic toxic features and the fact that a clear history of mercury exposure is generally not voluntarily provided by patients, M-MCD is highly likely to be misdiagnosed as primary minimal change disease (P-MCD) and treated with immunosuppressive therapy in clinical practice.

Confirming the definite diagnosis of M-MCD is crucial for proper treatment. As shown in the literature, most patients with M-MCD are highly responsive to mercury detoxification monotherapy and could even achieve complete remission $[5,6,10-13]$. Thus, the inappropriate treatment of corticosteroids and immunosuppressive agents could be avoided. However, understanding of MMCD is still insufficient, and most studies in this area are in the form of case reports. To date, the difference between M-MCD and P-MCD has not yet well known, and a well-designed comparison among such patients has not been conducted. In this study, we aimed to find key characteristics of M-MCD and facilitate differential diagnosis, by conducting retrospective comparison between $\mathrm{M}$ MCD and P-MCD.

\section{Materials and Methods}

\section{Patient Recruitment}

Twenty-one patients with renal biopsy proven M-MCD during January 2010 and December 2015 in Peking University First Hospital with a follow-up longer than 2 years were retrospectively recruited. Patients who met the following criteria are eligible: (1) older than 18 years, (2) had a definite contact history of mercurycontaining compounds, whose serum concentration of mercury was $>2.5 \mu \mathrm{g} / \mathrm{L}$ and spot urinary concentration of mercury $>10$ $\mu \mathrm{g} / \mathrm{L}$, and (3) the histological changes of patients were consistent with those of MCD. Patients with other known renal diseases were excluded. Twenty-one patients with P-MCD who were in the same age range (21-50 years) as the patients with M-MCD and those who had a mercury concentration of $<2.5 \mu \mathrm{g} / \mathrm{L}$ in blood and spot urine samples were recruited as the control group. The definition of mercury intoxication was based on a urinary mercury concentration of $>10 \mu \mathrm{g} / \mathrm{L}(50 \mathrm{nmol} / \mathrm{L})$ in spot sample, in compliance with the national standard of occupational mercury poisoning (GBZ892002) issued by the government of China. Clinical and laboratory data were recorded on admission as well as during the follow-up.

Quantification of Mercury

Being the same as reported previously [14], the quantification of mercury in blood and urine was detected using the inductively coupled plasma mass spectrometry (ICP-MS). The normal reference value in blood was $<2.5 \mu \mathrm{g} / \mathrm{L}(12.5 \mathrm{nmol} / \mathrm{L})$.

\section{Renal Pathological Scoring}

Renal specimens were processed by routine direct immunofluorescence (IF) on frozen tissue, light microscopy, and electron microscopy (EM), in accordance with the standard procedure in Peking University First Hospital [15]. For light microscopy, all cases were stained with hematoxylin and eosin, periodic acid-Schiff, silver methenamine (Meth), and Masson's trichrome. The proportion of globally sclerosed glomeruli, crescent formation, tubular injury with naked tubular basement membrane, and renal arteriolar atherosclerosis was estimated, respectively. Vacuolization of tubular epithelial cell was classified as 4 scales according to the severity: 0 for none, 1 for mild, 2 for medium, and 3 for heavy. Semiquantitative scores for classifying other tubular injuries (tubular brush border loss and tubular atrophy) and interstitial injuries (fibrosis and inflammation) were referred to the criteria of the Banff working classification of a $0-4$ scale as follows: 0 for no lesion, 1 for levels of parenchyma affected by the lesion $\leq 25 \%, 2$ for levels between 25 and 50\%, 3 for levels between 50 and $75 \%$, and 4 for levels $\geq 75 \%[16,17]$. The renal tissues were reviewed by 2 experienced renal pathologists independently. Differences of diagnosis or scoring between them were resolved by re-reviewing the biopsies and reaching a consensus.

\section{Measurement of Podocyte Foot Process Width}

The preparation of the renal biopsies for EM was performed following normal procedures described previously [18]. Photos of about 15-20 areas of 1-2 glomeruli in each case were taken randomly, with a magnification rate of 10,000 times. Measurements were made by 2 experienced renal pathologists separately. Any differences in measurement between the 2 were resolved by re-measuring the specimen and reaching a consensus.

Foot process width (FPW) was used to assess the podocyte effacement. For each EM photograph, the glomerular basement membrane (GBM) was traced and measured with standard image processing (Image-Pro Plus 6.0). The number of podocyte foot processes along the GBM was counted manually. The foot process was defined as any connected epithelial segment butting on the basement membrane, between 2 neighboring filtration pores or slits [19]. Measurements were made only in capillary loops. Kidney tissues from kidney transplant donors were used as normal controls. For each patient, the arithmetic mean of the FPW was calculated as follows:

$$
\mathrm{FPW}=\pi / 4 \cdot \Sigma \text { GBM length } / \Sigma \text { foot process }
$$

where $\Sigma$ GBM length represents the total GBM length counted in pictures of each patient and $\Sigma$ foot process represents the total number of foot processes counted in pictures of each patient. The 
Table 1. Demographic data, etiology of patients with M-MCD

\begin{tabular}{|c|c|c|c|c|c|c|}
\hline Cases & Gender & $\begin{array}{l}\text { Age, } \\
\text { years }\end{array}$ & Mercury exposure & $\begin{array}{l}\text { Duration of mercury } \\
\text { exposure, months }\end{array}$ & $\begin{array}{l}\text { Urinary mercury } \\
\text { concentration, } \mu \mathrm{g} / \mathrm{L}\end{array}$ & $\begin{array}{l}\text { Duration of renal } \\
\text { symptoms, months }\end{array}$ \\
\hline 1 & $\mathrm{~F}$ & 27 & Mercury-containing pills & 3.0 & 13.6 & 0.67 \\
\hline 3 & $\mathrm{~F}$ & 35 & Skin-lightning cosmetic & 5.0 & 20.2 & 1.00 \\
\hline 4 & $\mathrm{~F}$ & 49 & Mercury-containing pills & 4.0 & 23.1 & 0.50 \\
\hline 5 & $\mathrm{~F}$ & 41 & Skin-lightning cosmetic & 6.0 & 25.1 & 0.50 \\
\hline 8 & $\mathrm{~F}$ & 39 & Skin-lightning cosmetic & 12.0 & 36.9 & 12.00 \\
\hline 9 & $\mathrm{~F}$ & 36 & Skin-lightning cosmetic & 120.0 & 29.0 & 0.33 \\
\hline 10 & $\mathrm{~F}$ & 34 & Skin-lightning cosmetic & 5.0 & 29.5 & 0.67 \\
\hline 11 & $\mathrm{~F}$ & 48 & Mercury-containing pills & 1.5 & 29.8 & 0.83 \\
\hline 12 & $\mathrm{~F}$ & 30 & Hair-dyeing agents & 72.0 & 34.5 & 1.00 \\
\hline 17 & $\mathrm{M}$ & 50 & Mercury-containing pills & NA & 53.6 & 4.00 \\
\hline 18 & $\mathrm{~F}$ & 26 & Skin-lightning cosmetic & 1.0 & 54.3 & 0.30 \\
\hline 19 & $\mathrm{~F}$ & 39 & Skin-lightning cosmetic & 1.0 & 71.0 & 0.50 \\
\hline 20 & $\mathrm{~F}$ & 32 & Skin-lightning cosmetic & 10.0 & 73.0 & 1.50 \\
\hline 21 & $\mathrm{~F}$ & 43 & Mercury-containing pills & 3.0 & 130.1 & 4.00 \\
\hline
\end{tabular}

M-MCD, mercury-associated minimal change disease; NA, not applicable.

correction factor, $\pi / 4$, serves to adjust for presumed random variation in the angle of sections relative to the long axis of the podocyte [20]. At least $400 \mu \mathrm{m}$ GBM in length were measured for each case.

\section{Treatment}

The primary treatment for patients with M-MCD included exposure cessation and mercury detoxification therapy. Corticosteroids were prescribed for patients irresponsive to chelation therapy. The specific regimen of mercury detoxification was performed following the method described previously $[10,21]$. The regimen continued until the urine mercury level declined to the normal range, or a presence of any severe side effect.

Patients with P-MCD received conventional therapy according to the Kidney Disease: Improving Global Outcomes guideline for glomerulonephritis in 2012 [22]. The criteria of renal outcome were defined as follows [22]: (1) complete remission: reduction of proteinuria to $<0.3 \mathrm{~g} /$ day, stable serum $\mathrm{Cr}$ and serum albumin $>3.5$ $\mathrm{g} / \mathrm{dL}$, with the symptom improved remarkably; (2) partial remission: reduction of proteinuria to $0.3-3.5 \mathrm{~g} /$ day and a decrease $>50 \%$ from baseline, with the symptoms improved; (3) treatment failure: reduction of proteinuria did not reach the criteria mentioned above, or no improvement in symptoms; (4) relapse: recurrence of proteinuria $>3.5 \mathrm{~g}$ /day after complete remission.

Statistical Analysis

Statistical analysis was performed with statistical software SPSS v20.0 (Chicago, IL, USA). The $\chi^{2}$ test or Fisher's exact test was used to compare categorical variables. Continuous variables with nor- mal distribution were expressed as the mean \pm SD and were compared using the independent-samples $t$ test. Continuous variables with non-normal distributions were presented as the median with interquartile range (IQR) and analyzed using the Mann-Whitney $\mathrm{U}$ test. Comparison between groups was performed using one-way ANOVA. The binary logistic regression model was performed to identify the predictor(s) which can differentiate M-MCD from PMCD. Next, the receiver operating characteristics (ROC) curve analysis was used to determine the optimum cutoff of FPW in differentiating M-MCD from P-MCD. The cutoff value we chose was the FPW corresponding to the point where the Youden's index took the maximum value. Statistical significance was recognized when $p<0.05$.

\section{Results}

Demographic Characteristics of Patients with M-MCD

The demographic data of the 21 patients with M-MCD are shown in Table 1 . Two patients were male, 19 were female, with an average age of $35.1 \pm 8.2$ years at diagnosis. The average duration of mercury exposure before the occurrence of proteinuria was 4.0 (IQR 2.5-8.0) months. The urinary mercury concentrations ranged from 13.6 to $130.1 \mu \mathrm{g} / \mathrm{L}$ (5-52 times higher than the reference value for the general population). Mercury exposure was caused by 
Table 2. Comparison of clinical data and prognosis between patients with M-MCD and P-MCD

\begin{tabular}{lccc}
\hline Parameters & M-MCD $(n=21)$ & P-MCD $(n=21)$ & $p$ value \\
\hline Gender, male/female & $19 / 2$ & $15 / 6$ & 0.454 \\
Age, years & $35.1 \pm 8.2$ & $35.4 \pm 11.2$ & 0.938 \\
Duration of renal symptoms, months & $0.83,0.5-1.5$ & $0.86,0.5-2.0$ & 0.970 \\
Nephrotic syndrome, $n / N(\%)$ & $18 / 21(85.7)$ & $21 / 21(100)$ & 0.232 \\
Edema, $n / N(\%)$ & $19 / 21(90.5)$ & $16 / 21(76.2)$ & 0.410 \\
Hypertension, $n / N(\%)$ & $3 / 21(14.3)$ & $2 / 21(9.5)$ & 1.000 \\
Urinary protein, g/24 h & $5.8 \pm 4.5$ & $6.6 \pm 4.4$ & 0.576 \\
Microscopic hematuria, $n / N(\%)$ & $4 / 21(19.0)$ & $5 / 21(23.8)$ & 1.000 \\
Albumin, g/L & $18.5,16.6-20.9$ & $20.3,16.7-24.1$ & 0.624 \\
Serum Cr, $\mu$ mol/L & $63.0,56.0-70.0$ & $72.3,54.5-79.0$ & 0.302 \\
BUN, mmol/L & $4.2,3.7-4.9$ & $4.8,3.9-6.1$ & 0.083 \\
Hemoglobin, g/L & $137.4 \pm 21.1$ & $141.1 \pm 20.9$ & 0.580 \\
Cholesterol, mmol/L & $8.9 \pm 2.4$ & $12.5 \pm 4.1$ & 0.002 \\
ANA $\geq 1: 100, n / N(\%)$ & $4 / 21(19.0)$ & $3 / 20(15.0)$ & 1.000 \\
IgG, g/L & $4.6,3.3-7.5$ & $4.5,2.8-6.0$ & 0.550 \\
Urinary NAG enzyme, U/L & $113.0,30.0-180.0$ & $90.0,62.0-177.0$ & 0.475 \\
Urinary protein electrophoresis & & & 0.737 \\
$\quad$ Low molecular weight proteinuria, $\%$ & $0,0-1.4$ & $0.5,0-1.4$ & 0.459 \\
Albumin, \% & $74.1 \pm 7.2$ & $71.5 \pm 8.4$ & 0.441 \\
High molecular weight proteinuria, \% & $25.3 \pm 6.7$ & $27.8 \pm 8.2$ & 0.023 \\
Remission time, months & $2.0,1.0-4.0^{\mathrm{a}}$ & $1.0,0.5-1.8^{\mathrm{b}}$ & 0.003 \\
Relapse, $n / N(\%)$ & $0 / 16^{\mathrm{a}}(0 \%)$ & $8 / 17^{\mathrm{b}}(47.1 \%)$ \\
\hline
\end{tabular}

Data are presented as $n / N(\%)$, mean $\pm \mathrm{SD}$, or median (25th-75th percentile). M-MCD, mercury-associated minimal change disease; P-MCD, primary minimal change disease; ANA, antinuclear antibody; NAG, N-Acetyl$\beta$-glucosaminase; IgG, immunoglobulin G; Remission time, the time from initial treatment to complete remission. ${ }^{\mathrm{a}} n=16$ (5 patients lost in follow-up). ${ }^{\mathrm{b}} n=17$ (4 patients did not achieve complete remission).

the use of skin-lightening cosmetics for 14 patients (66.7\%), mercury-containing pills for 5 patients (23.8\%), hair-dyeing agents for 1 patient (4.8\%), and unidentified reason for 1 patient (4.8\%). Mercury was detected in daily-use cosmetic for 1 patient, and the mercury concentration was $19,601 \mathrm{mg} / \mathrm{kg}$, substantially higher than the upper limit of national standard for cosmetics, which is 1 $\mathrm{mg} / \mathrm{kg}$.

\section{Comparison of Clinical Data between Patients with $M-M C D$ and $P-M C D$}

The clinical characteristics in both groups of patients are shown in Table 2. There were no significant differences between patients with M-MCD and P-MCD for major clinical parameters, including edema, hypertension, urinary protein, hematuria, albumin, serum $\mathrm{Cr}$, etc.

\section{Pathological Parameters}

Findings on kidney biopsy of M-MCD are shown in Figure 1. The glomerulus was generally normal (Fig. 1a). As shown in Table 3, the percentage of patients with sclerotic lesions in glomeruli was similar between patients with M-MCD and P-MCD (0.0 [IQR 0.0-0.95\%] vs. 0.0 [IQR $0.0-0.95 \%$ ], $p=0.107)$. For $20 \mathrm{M}-\mathrm{MCD}$ patients available for reliable renal pathological scoring, mild vacuolization of renal tubular epithelial cells was observed for 17 patients (85\%) (Fig. 1b). Tubular brush border loss was observed for 4 patients (20\%) (Fig. 1c). One patient had naked renal tubule formation and 1 with renal tubular atrophy. Two patients presented with interstitial inflammatory cell infiltration (Fig. 1d). Nine patients (45\%) presented with arteriolosclerosis of renal arterioles. The tubulointerstitial injury for patients with M-MCD was generally mild, and there was no significant difference in pathological parameters between the 2 groups.

\section{EM and Morphometry}

Representative pictures are shown in Figure 2. The average FPW in the normal control group was $506.1 \pm 44.9$ $\mathrm{nm}$, which is in accordance with the normal range described by our center [23] and others [24]. 

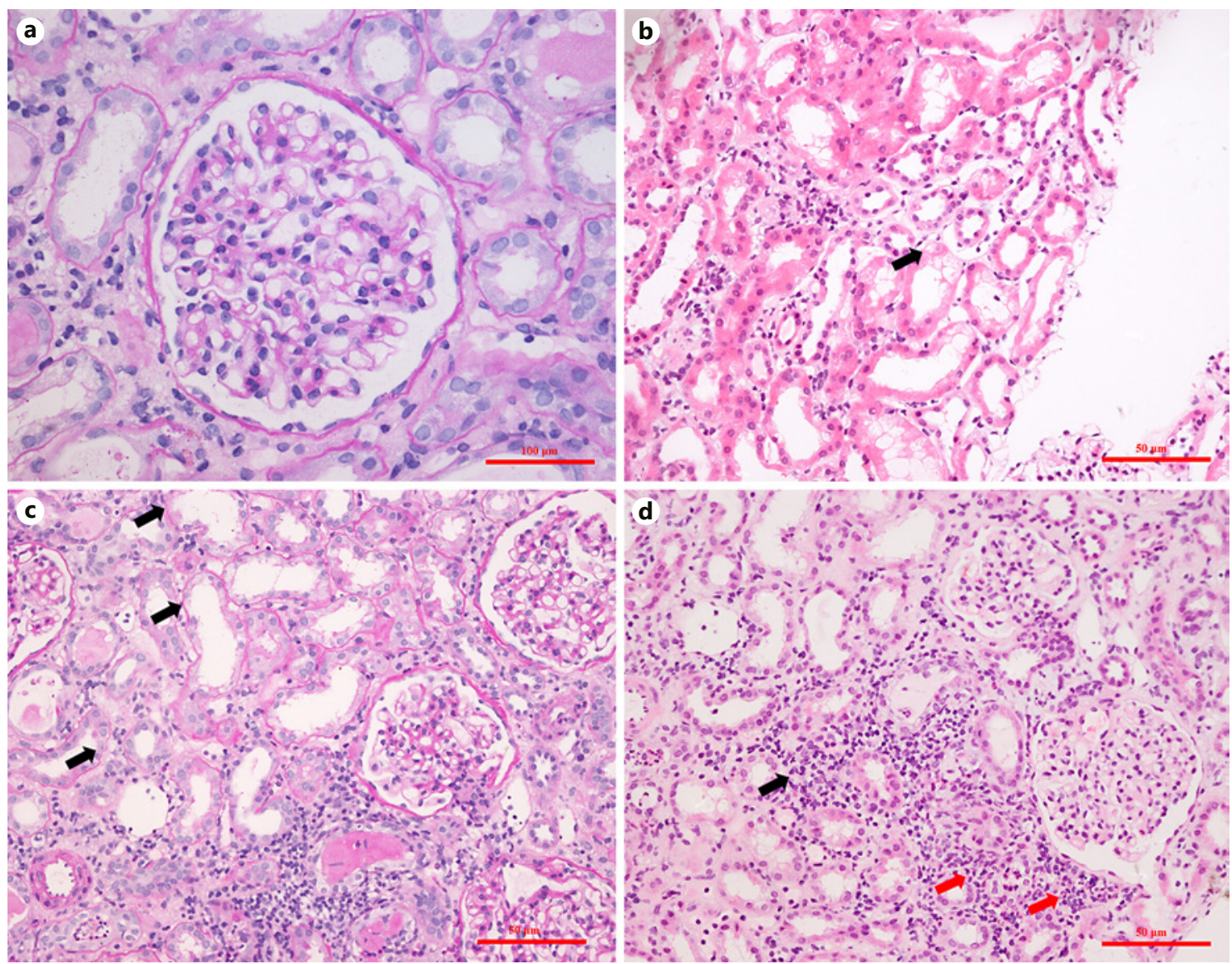

Fig. 1. Findings on kidney biopsy of M-MCD. a The glomerulus was generally normal (Patient \#3 in Table 1, PAS $\times 400$ ). b Vacuolization of renal tubular epithelial (arrow) (Patient \#14 in Table 1, HE $\times 200$ ). c Tubular brush border loss (arrow) (Patient \#3 in Table 1, PAS $\times 200$ ). d Interstitial inflammatory infiltration (the black arrow indicates lymphoid and monocyte infiltration and the red arrow indicates neutrophil infiltration) (Patient \#3 in Table 1, HE ×200).M-MCD, mercury-associated minimal change disease; HE, hematoxylin and eosin.

As shown in Figure 3, the FPW values of patients with M-MCD and P-MCD were significantly higher than that of normal controls $(p<0.001)$. Patients with M-MCD had significantly lower levels of FPW than those with P-MCD (935.0 [IQR 853.7-1,176.7] nm vs. 1,403.2 [IQR 1,089.2$1,841.8] \mathrm{nm}, p=0.004)$.

As there were no significant differences between $\mathrm{M}$ MCD and P-MCD in clinical or routine pathological variables by univariate analysis, variables with potential clinical significance, including gender, serum albumin, serum $\mathrm{Cr}$, and proteinuria, together with FPW were put into the binary logistic regression model to do the analysis. And the results showed that FPW was an independent predictor to differentiate M-MCD from P-MCD ( $B=$ -0.003 , Wald $\chi^{2}=4.562, p=0.033$, OR $=0.997$ ).
We then generated an ROC curve to investigate the diagnostic ability of FPW (Fig. 4), which revealed an AUC of 0.787 . Patients with M-MCD can be characterized by an FPW below $1,385 \mathrm{~nm}$ with a sensitivity of $94.7 \%$ and a specificity of $52.4 \%$.

\section{Treatment and Outcomes}

All 21 patients with M-MCD stopped mercury exposure after diagnosis. Other treatments included mercury detoxification and supportive therapy. Nine out of $21 \mathrm{pa}-$ tients did not receive corticosteroids or immunosuppressive agent before mercury detoxification. Among them, 7 patients received 4.0 (IQR 2.0-5.0) rounds of mercury detoxification with sodium dimercaptosulfonate and achieved complete remission in 2.0 (IQR 0.63-6.0) 
Table 3. Comparison of pathological parameters between patients with M-MCD and P-MCD

\begin{tabular}{|c|c|c|c|c|}
\hline Parameters & Grade & $\operatorname{M-MCD}\left(n=20^{\mathrm{a}}\right)$ & $\mathrm{P}-\mathrm{MCD}(n=21)$ & $p$ value \\
\hline \multicolumn{5}{|l|}{ Glomerular lesion } \\
\hline \multicolumn{2}{|l|}{ Sclerosis in glomeruli, \% } & $0.0,0.0-0.95$ & $0.0,0.0-0.0$ & 0.107 \\
\hline \multicolumn{2}{|l|}{ Crescent formation, $n(\%)$} & $0(0)$ & $0(0)$ & 1.000 \\
\hline \multicolumn{5}{|l|}{ Tubulointerstitial lesion } \\
\hline \multirow[t]{4}{*}{ PTEC vacuolization, $n(\%)$} & 0 & $3(15)$ & $2(9.5)$ & 0.229 \\
\hline & 1 & $17(85)$ & $17(81)$ & \\
\hline & 2 & $0(0)$ & $2(9.5)$ & \\
\hline & 3 & $0(0)$ & $0(0)$ & \\
\hline \multirow[t]{5}{*}{ Tubular brush border loss, $n(\%)$} & 0 & $16(80)$ & $16(76)$ & 0.393 \\
\hline & 1 & $3(15)$ & $5(24)$ & \\
\hline & 2 & $0(0)$ & $0(0)$ & \\
\hline & 3 & $1(5)$ & $0(0)$ & \\
\hline & 4 & $0(0)$ & $0(0)$ & \\
\hline \multicolumn{2}{|l|}{ Naked TBM, $n(\%)$} & $1(5)$ & $0(0)$ & 0.488 \\
\hline \multirow[t]{5}{*}{ Tubular atrophy, $n(\%)$} & 0 & $19(95)$ & $19(90)$ & 1.000 \\
\hline & 1 & $1(5)$ & $2(10)$ & \\
\hline & 2 & $0(0)$ & $0(0)$ & \\
\hline & 3 & $0(0)$ & $0(0)$ & \\
\hline & 4 & $0(0)$ & $0(0)$ & \\
\hline \multirow[t]{5}{*}{ Interstitial fibrosis, $n(\%)$} & 0 & $20(100)$ & $20(95)$ & 1.000 \\
\hline & 1 & $0(0)$ & $1(5)$ & \\
\hline & 2 & $0(0)$ & $0(0)$ & \\
\hline & 3 & $0(0)$ & $0(0)$ & \\
\hline & 4 & $0(0)$ & $0(0)$ & \\
\hline \multirow[t]{5}{*}{ Interstitial inflammatory infiltration, $n(\%)$} & 0 & $18(90)$ & $21(100)$ & 0.488 \\
\hline & 1 & $1(5)$ & $0(0)$ & \\
\hline & 2 & $0(0)$ & $0(0)$ & \\
\hline & 3 & $1(5)$ & $0(0)$ & \\
\hline & 4 & $0(0)$ & $0(0)$ & \\
\hline \multicolumn{5}{|l|}{ Renal arteriole lesion } \\
\hline \multicolumn{2}{|l|}{ Renal arteriolar atherosclerosis, $n(\%)$} & $9(45)$ & $9(43)$ & 1.000 \\
\hline
\end{tabular}

months, while the other 2 patients showed treatment failure. The complete remission rate of mercury detoxification monotherapy was $77.8 \%$ (7/9). With treatment of corticosteroids, the other 2 patients, who were irresponsive to detoxification regimen, achieved complete remission in 2 and 7 months, respectively.

In contrary, 7 out of $21 \mathrm{M}-\mathrm{MCD}$ patients initially received immunosuppressive therapy before the diagnosis and received detoxification therapy thereafter. They received 3.0 [(IQR 2.5-4.5] rounds of mercury detoxification with dimercaptosulfonate and achieved complete remission in 2.0 [IQR 1.5-2.5] months. However, the rest 5 among the 21 patients were lost in follow-up after discharge.

For 21 patients with $\mathrm{P}-\mathrm{MCD}, 17$ achieved complete remission in 1.0 [IQR $0.5-1.8$ ] months after initial treatment and $47.1 \%(8 / 17)$ of them relapsed, 1 patient achieved partial remission, and the other 3 patients were treatment failure. The prognosis of patients with M-MCD and P-MCD was also compared, as shown in Table 2. The median time from initial treatment to complete remis- 

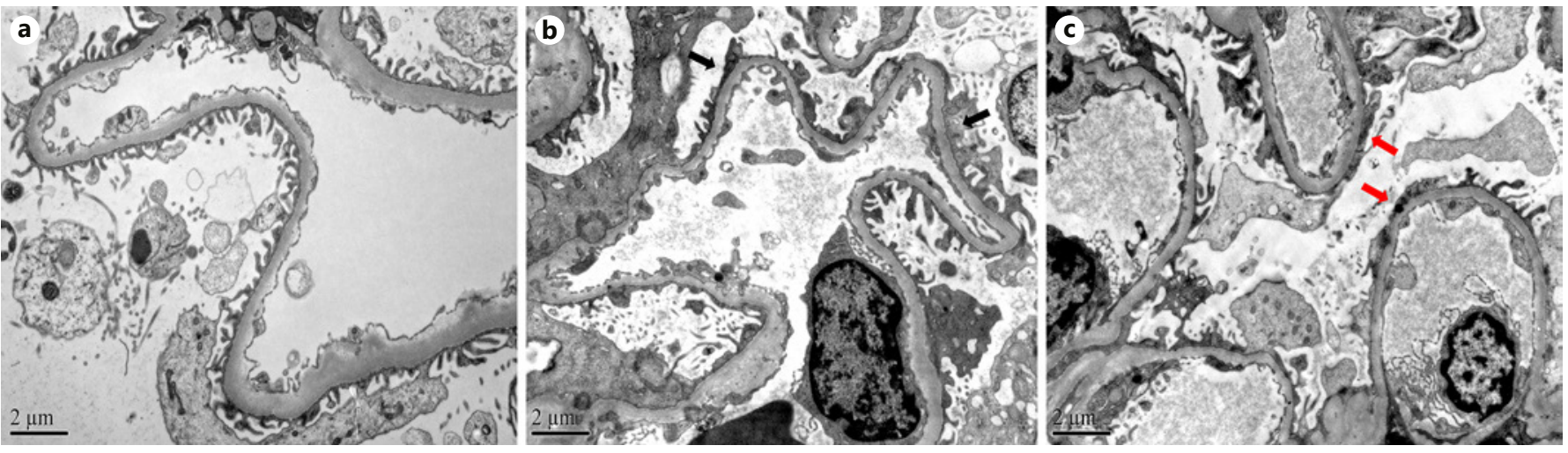

Fig. 2. EM to illustrate the ultrastructural features of M-MCD (original magnification, $\times 10,000$ ). a Example of normal control. Kidney tissue from kidney transplant donor. The foot processes were separate. $\mathbf{b}$ Example of M-MCD. Normal GBM, no immune deposits, and partial effacement of foot processes (black arrows). c Example of P-MCD. Extensive effacement of foot processes (red arrows). M-MCD, mercury-associated minimal change disease; $\mathrm{P}$ MCD, primary minimal change disease; EM, electron microscopy; GBM, glomerular basement membrane.
Fig. 3. FPW of patients with M-MCD, P$\mathrm{MCD}$, and normal control. Data expressed as median with range (IQR). M-MCD, mercury-associated minimal change disease; P-MCD, primary minimal change disease; FPW, foot process width; IQR, interquartile range.

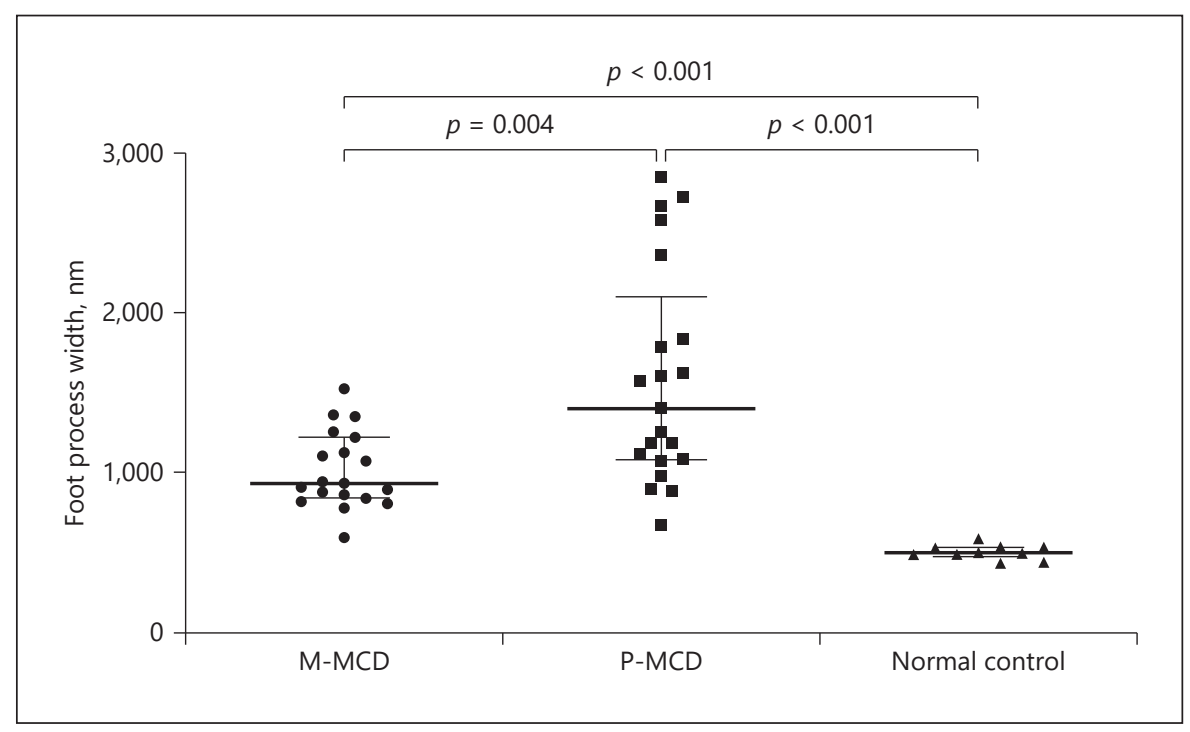

sion was longer for patients with M-MCD than patients with P-MCD (2.0 [IQR 1.0-4.0] months vs. 1.0 [IQR 0.51.8 ] months, $p=0.023)$. However, no patients with $\mathrm{M}$ MCD relapsed after complete remission, while nearly half of patients with P-MCD relapsed, with a significant difference ( 0 vs. $47.1 \%, p=0.003$ ).

To evaluate the role of immunosuppressants in the treatment of $\mathrm{M}-\mathrm{MCD}$, we compared the time required to achieve complete remission between patients treated with mercury detoxification monotherapy and patients initially treated with immunosuppressive therapy. There was no significant difference in remission time between the 2 groups (2.0 [IQR 1.0-6.0] months vs. 2.0 [IQR 1.5-2.5] months, $p=0.606)$.

\section{Discussion}

MN has been generally believed to be the main pathological type of mercury-associated glomerulopathy [9]. However, proven MCD caused by mercury-containing skin-lightening cream raised more attention in recent 


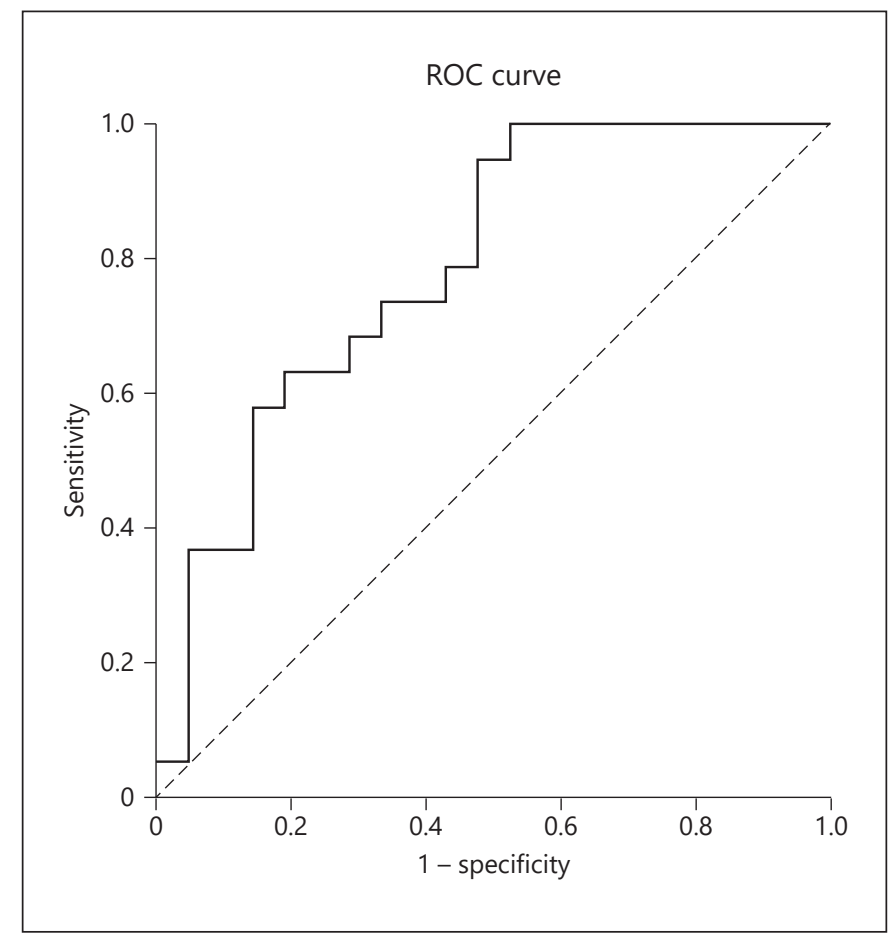

Fig. 4. ROC curve to investigate the diagnostic ability of FPW. FPW, foot process width; ROC, receiver operating characteristics.

years after being first reported by Tang et al. [11]. In our previous study, we found that MCD (accounting for $60 \%$ ), rather than $\mathrm{MN}$ (accounting for $37.1 \%$ ), was the most common pathological type [10], which is in line with a recent review by Chan et al. [6], which reported that among patients with exposure to mercury-containing skin-lightening creams, $67 \%$ revealed MCD and only $23 \%$ revealed MN. While in the current study, we further enlarged the case numbers and implemented a comprehensive comparison of clinical features between M-MCD and P-MCD. To the best of our knowledge, this is the largest case series of M-MCD and is the first one to compare the clinicopathological manifestations and ultrastructural characteristics between patients with M-MCD and P-MCD.

The diagnosis of mercury poisoning is based primarily on exposure to mercury-containing compounds, and supported by elevated urinary mercury concentrations. In this study, the exposure of mercury mainly came from mercury-containing skin-lightening cosmetics, followed by mercury-containing pills and hair-dyeing agents. All patients with M-MCD in this study had mercury exposure before the presence of proteinuria, but none of them had any evidence of other renal diseases. The main find- ing was that the urinary mercury concentrations of these patients were markedly increased, especially for those who use cosmetics with mercury content much higher than the national upper limit. Moreover, after receiving mercury detoxication monotherapy, most patients achieved complete remission. These findings suggested that it was chronic mercury poisoning that caused the MCD.

The average age of patients with M-MCD in the current study was 35.1 years, which is not the common age when MCD occurs. Besides, the majority of the patients were females; this is because the primary cause of mercury poisoning is the use of skin-lightening cosmetics. In this case, even though the history is not provided voluntarily by patients, mercury exposure should be considered for young and mid-age females with nephrotic syndrome, especially for those whose facial skin is notably lighter than that of other body parts.

In this study, we found that clinical and routine pathological features were similar for patients with M-MCD and P-MCD, especially for the finding of mild tubulointerstitial injury, and it is consistent with previous studies $[4,21]$. However, differences in ultrastructural features were detected between the 2 groups. It is well known that podocyte foot process effacement (FPE) is a typical feature in all types of nephrotic syndrome. Although the exact mechanism resulting in FPE remains unknown $[25,26]$, it is widely considered that it could be a stereotypic reaction of podocytes to damages [27]. It is suggested that the extent of FPE might depend on the underlying disease and be determined by the nature of podocyte damage [28]. Therefore, the degree of FPE may be used to distinguish different pathogenic factors and underlying diseases. As a kind of podocytopathy, MCD was classified into several categories according to different podocyte damages. The mechanism by which mercury causes MCD has not been elucidated. Eichler et al. [29] speculated that mercury exposure may induce glomerular injury in part via induction of podocyte apoptosis. In this study, we found the FPE in patients with M-MCD was less severe than in those with P-MCD. The different morphological changes of foot process may suggest that the pathogenesis of the 2 diseases may be different. According to our ROC analysis, a cutoff value of $1,385 \mathrm{~nm}$ for FPW might differentiate patients with M-MCD from P-MCD with high sensitivity, but the specificity was suboptimal, inducing a large overlap of the interquartile range of the 2 types of MCD. Thus, it may be premature to use $1,385 \mathrm{~nm}$ as a cutoff to make a differentiation, but based on all of the information we have, we think it 
should be a meaningful value for renal pathologists' reference to alert the possibility of mercury poisoning in MCD patients with partial effacement of foot processes. Further studies with larger sample size are needed to validate the ability of FPW in differentiating M-MCD from P-MCD.

Mercury detoxification is effective for M-MCD treatment. In previous studies, researchers found that some patients could achieve complete remission with detoxification monotherapy $[5,11,13]$. Being consistent with those findings, in this study, we found that $77.8 \%$ of patients with M-MCD achieved complete remission after detoxification monotherapy, and none of them relapsed, which confirmed the critical role of detoxification therapy. Moreover, we found the remission time of patients who initially received immunosuppressive treatment before detoxification therapy was not shorter than those who received detoxification monotherapy. This finding suggested that immunosuppressive therapy did not shorten the remission time for M-MCD patients.

However, there are some limitations in the present study. First, given that this is a retrospective study with a small sample size, the significance of the findings might be limited, which should be taken with caution and requires validation from studies with a larger cohort. Second, spot urine sample for mercury concentration measurement was performed in this study. Although it was found that spot urine mercury concentrations correlated fairly well with that of 24-h urine [30], a 24-h urine mercury measurement might be more reliable for long-term mercury exposure. Third, the distribution of different types of mercury exposures in different geographic regions is important. As all patients lived in inland cities in our study, there seems to be no significant difference in geographic regions among these patients. Further explorations in prospective multicenter studies are needed. Fourth, in the ultrastructural measurement by EM, only 1-2 glomeruli in each patient were inspected, which may cause potential bias. Last, the follow-up intervals were longer than the actual remission time after the treatment for some patients, and some patients were lost in followup, so the results may underestimate the effectiveness of detoxification therapy.

In conclusion, we found that M-MCD patients showed similar clinicopathological features with P-MCD patients, but with less severe FPE, suggesting different pathogenesis of these 2 disease entities. The treatment of mercury detoxification was highly effective for patients with M-MCD and can be considered as a primary choice in clinical practice.

\section{Acknowledgements}

We appreciate Li-jun Chai and Ya-li Ren from the department of Electron Microscopy Laboratory, Peking University First Hospital, for providing technical support in glomeruli photograph and podocyte foot process width measurement.

\section{Statement of Ethics}

Written informed consent was obtained from each patient for renal tissue, blood, and urine sampling. The research was in compliance with the Declaration of Helsinki and was approved by the ethics committee of Peking University First Hospital (approval number 2017[1280]).

\section{Conflict of Interest Statement}

The authors have no conflicts of interest to declare.

\section{Funding Sources}

This work was supported by Chinese Academy of Medical Sciences Research Unit (No. 2019RU023), Peking University, and CAMS Innovation Fund for Medical Sciences (2019-I2M-5-046).

\section{Author Contributions}

A.b.Q. collected the data, interpreted the statistical results, and wrote the first draft. F.d.Z. and M.h.Z. contributed to the design of the protocol and revised the manuscript. X.j.Y. performed the renal pathological scoring. S.x.W. performed the glomeruli photograph and podocyte foot process width measurement. All authors read and approved the final manuscript.

References

1 George CR. Mercury and the kidney. J Nephrol. 2011;24(Suppl 17):S126-32.

2 Ye BJ, Kim BG, Jeon MJ, Kim SY, Kim HC, Jang TW, et al. Evaluation of mercury exposure level, clinical diagnosis and treatment for mercury intoxication. Ann Occup Environ Med. 2016;28:5.

3 Cameron JS, Trounce JR. Membranous glomerulonephritis and the nephrotic syndrome appearing during mersalyl therapy. Guys Hosp Rep. 1965;114:101-7.

4 Wang YY, Cheng H, Wang GQ, Dong HR, Chen YP, Wang CJ, et al. Minimal change disease due to mercury-containing skin lightening cream [abstract]. Nephrol Dial Transplant. 2013;28:1394-5.

5 Tang HL, Mak YF, Chu KH, Lee W, Fung SK, Chan TY, et al. Minimal change disease caused by exposure to mercury-containing skin lightening cream: a report of 4 cases. Clin Nephrol. 2013;79(4):326-9. 
6 Chan TYK, Chan APL, Tang HL. Nephrotic syndrome caused by exposures to skin-lightening cosmetic products containing inorganic mercury. Clin Toxicol. 2020;58(1):9-15.

7 Li SJ, Zhang SH, Chen HP, Zeng CH, Zheng CX, Li LS, et al. Mercury-induced membranous nephropathy: clinical and pathological features. Clin J Am Soc Nephrol. 2010;5(3): 439-44.

8 Doshi M, Annigeri RA, Kowdle PC, Subba Rao B, Varman M. Membranous nephropathy due to chronic mercury poisoning from traditional Indian medicines: report of five cases. Clin Kidney J. 2019;12(2):239-44.

9 Miller S, Pallan S, Gangji AS, Lukic D, Clase CM. Mercury-associated nephrotic syndrome: a case report and systematic review of the literature. Am J Kidney Dis. 2013;62(1): $135-8$.

10 Qin $\mathrm{AB}$, Su T, Wang SX, Zhang F, Zhou FD, Zhao MH. Mercury-associated glomerulonephritis: a retrospective study of 35 cases in a single Chinese center. BMC Nephrol. 2019; 20(1):228.

11 Tang HL, Chu KH, Mak YF, Lee W, Cheuk A, Yim KF, et al. Minimal change disease following exposure to mercury-containing skin lightening cream. Hong Kong Med J. 2006; 12(4):316-8.

12 Su T, Liu XL, Zhang YM, Mao WB, Liu G, Wang SX, et al. [Clinicopathological analysis of mercury poisoning-associated glomerulonephropathy]. Chin J Nephrol. 2011;27(5): 333-6.

13 Choi J, Cheung T, Choi T. Mercury poisoning caused by use of a facial cream. Public Health Epidemiol Bull. 2002;11:49-52.

14 Taylor CM, Golding J, Emond AM. Blood mercury levels and fish consumption in pregnancy: risks and benefits for birth outcomes in a prospective observational birth cohort. Int J Hyg Environ Health. 2016;219(6):51320.
15 Huang J, Liu G, Zhang YM, Cui Z, Wang F, Liu XJ, et al. Plasma soluble urokinase receptor levels are increased but do not distinguish primary from secondary focal segmental glomerulosclerosis. Kidney Int. 2013;84(2):36672.

16 Racusen LC, Solez K, Colvin RB, Bonsib SM, Castro MC, Cavallo T, et al. The Banff 97 working classification of renal allograft pathology. Kidney Int. 1999;55(2):713-23.

17 Solez K, Colvin RB, Racusen LC, Haas M, Sis $\mathrm{B}$, Mengel M, et al. Banff 07 classification of renal allograft pathology: updates and future directions. Am J Transplant. 2008;8(4):75360.

18 Liu XJ, Zhang YM, Wang SX, Liu G. Ultrastructural changes of podocyte foot processes during the remission phase of minimal change disease of human kidney. Nephrology. 2014;19(7):392-7.

19 van den Berg JG, van den Bergh Weerman MA, Assmann KJ, Weening JJ, Florquin S. Podocyte foot process effacement is not correlated with the level of proteinuria in human glomerulopathies. Kidney Int. 2004;66(5): 1901-6.

20 Gundersen HJ, Seefeldt T, Osterby R. Glomerular epithelial foot processes in normal man and rats. Distribution of true width and its intra- and inter-individual variation. Cell Tissue Res. 1980;205(1):147-55.

21 Zhang L, Liu F, Peng Y, Sun L, Chen C. Nephrotic syndrome of minimal change disease following exposure to mercury-containing skin-lightening cream. Ann Saudi Med. 2014; 34(3):257-61.
22 Cattran DC, Feehally J, Cook HT. Kidney disease improving global outcomes (KDIGO) glomerulonephritis work group. KDIGO clinical practice guideline for glomerulonephritis. Kidney Int. 2012;2(2):139-274.

23 Wang Y, Yu F, Song D, Wang SX, Zhao MH Podocyte involvement in lupus nephritis based on the 2003 ISN/RPS system: a large cohort study from a single centre. Rheumatology. 2014;53(7):1235-44.

24 Deegens JK, Dijkman HB, Borm GF, Steenbergen EJ, van den Berg JG, Weening JJ, et al. Podocyte foot process effacement as a diagnostic tool in focal segmental glomerulosclerosis. Kidney Int. 2008;74(12):1568-76.

25 Shankland SJ. The podocyte's response to injury: role in proteinuria and glomerulosclerosis. Kidney Int. 2006;69(12):2131-47.

26 Pavenstadt H, Kriz W, Kretzler M. Cell biology of the glomerular podocyte. Physiol Rev. 2003;83(1):253-307.

27 Kerjaschki D. Dysfunctions of cell biological mechanisms of visceral epithelial cell (podocytes) in glomerular diseases. Kidney Int. 1994;45(2):300-13.

28 Ahn W, Bomback AS. Approach to diagnosis and management of primary glomerular diseases due to podocytopathies in adults: core curriculum 2020. Am J Kidney Dis. 2020; 75(6):955-64.

29 Eichler T, Ma Q, Kelly C, Mishra J, Parikh S, Ransom RF, et al. Single and combination toxic metal exposures induce apoptosis in cultured murine podocytes exclusively via the extrinsic caspase 8 pathway. Toxicol Sci. 2006; 90(2):392-9.

30 Cianciola ME, Echeverria D, Martin MD, Aposian HV, Woods JS. Epidemiologic assessment of measures used to indicate lowlevel exposure to mercury vapor $(\mathrm{Hg})$. J Toxicol Environ Health. 1997;52(1):19-33. 